\title{
Resilient Tracking Control of Networked Control Systems Under Cyber Attacks
}

\author{
Eman Mousavinejad, Student Member, IEEE, Xiaohua Ge, Member, IEEE, Qing-Long Han, Fellow, IEEE, \\ Fuwen Yang, Senior Member, IEEE, and Ljubo Vlacic, Senior Member, IEEE
}

\begin{abstract}
This article is concerned with the resilient tracking control of a networked control system under cyber attacks. The attacker is an active adversary whose aim is to severely degrade the tracking performance of the system by launching deception attacks on the sensor-to-controller communication channels and denial-of-service attacks on the controller-to-plant channels, respectively. First, a concept of resilient set-membership tracking control is presented, through which the system's true state is guaranteed to reside in a bounding ellipsoidal set of the reference state regardless of the existence of attacks and unknown-butbounded (UBB) noises. Second, in the case that full information of the system's state is not implicitly trusted in the presence of attacks, a resilient set-membership estimation strategy is provided to secure the state estimates against the deception attacks. Furthermore, based on a recursive computation of a reference state ellipsoid and confidence state estimation ellipsoids, a convex optimization algorithm in terms of recursive linear matrix inequalities is proposed to obtain the gain parameters for both the desired resilient state estimator and the tracking controller. Finally, the effectiveness of the proposed method is illustrated through an Internet-based three-tank system.
\end{abstract}

Index Terms-Deception attack, denial-of-service (DoS) attack, networked control systems (NCSs), resilient tracking control, setmembership approach.

\section{INTRODUCTION}

$\mathbf{R}$ ECENT advancement and use of Internet, embedded systems, wireless communication technologies, and novel control strategies over the past few decades have significantly elevated the development and application of networked control systems (NCSs) in a wide range of practical fields, such as transportation systems, electrical power systems and smart grids, remote surgery, industrial and manufacturing systems, and so on [1]. Due to heterogeneous IT components and open network connections among controllers, sensors, actuators, and other networked components, the confidentiality, integrity, and availability (CIA) of exchanged data in an NCS may suffer from vulnerability to malicious cyber attacks. Undoubtedly, this kind of threat is mainly launched by an adversary in either the physical world or the cyberspace with the aim of substantial economic benefits or disrupting human life. Therefore, it is imperative to properly address security issues

Manuscript received April 04, 2018; revised August 11, 2019; accepted October 13, 2019. This work was supported by the Australian Research Council Discovery Project under Grant DP160103567. (Corresponding author: QingLong Han.)

E. Mousavinejad, F. Yang, and L. Vlacic are with the School of Engineering and Built Environment, Griffith University, Gold Coast, QLD 4222, Australia. (e-mails: eman.mousavinejad@griffithuni.edu.au; fuwen.yang@griffith.edu.au; L.Vlacic@griffith.edu.au)

X. Ge and Q.-L. Han are with the School of Software and Electrical Engineering, Swinburne University of Technology, Melbourne, VIC 3122, Australia. (e-mails: xge@swin.edu.au; qhan@swin.edu.au) of NCSs so as to ensure their reliable and safe performance [2], [3].

Note that NCSs have several distinct advantages, including flexible architectures and less installation and maintenance costs. The remote tracking control through a shared communication network has received an ever-increasing interest from researchers due to its extensive practical applications in industry and military, such as motor control [4], [5], robotic trajectory tracking control [6]-[8], and flight control [9]-[11]. Generally, the ultimate objective of tracking control is to provide the system with a proper control command such that the state (or output) of the system tracks a state (or output) trajectory predefined by a given reference model. In many realworld applications due to physical constraints, technological limitations, or even human safety aspects, it is an irrational approach to locate the physical plant, actuators, sensors, and controllers at the same place. Hence, in modern industrial systems, the system components are connected remotely via some wireless network medium.

Motivated by security concerns of NCSs, the property of the system's resiliency is of utmost significance. Generally speaking, resiliency refers to the ability of restoration of a system after being corrupted by unexpected adversarial attacks. Hence, a resilient tracking controller must be able, in the presence of a cyber attack, to recover its computational performance as well as to resume the system's operation which was in place just before the cyber attack. Up to date, cyber attacks triggered through unreliable communication networks can be arguably categorized into two main types: 1) denial-of-service (DoS) attacks and 2) deception attacks. DoS attacks are aimed at deteriorating the availability of the system information, usually either control commands or sensor data, by jamming the communication channels and therefore, preventing information exchange between components of NCSs. Technologically, DoS attacks can be implemented by disrupting the radio frequencies on wireless communication channels, which results in channel congestion. Since an adversary requires little prior knowledge about the system and does not need any special devices to launch a DoS attack, this type of attack can seriously threaten the NCS's performance. On the other hand, deception attacks represent a kind of attempt to violate the confidentiality and integrity of system information. Through the deception attacks, an adversary tries to manipulate the system into following his or her desired behavior via accessing disclosure resources and injecting deception information into either sensor data or control commands. For example, radars in a target tracking system can be deceived by deception attacks if the adversary successfully injects the deception signals into frequency- 
shifted copies of the radar's signals and hence, degrades the tracking performance [12].

During the past two decades, the problem of guaranteeing resilient NCSs has attracted considerable interest from different perspectives. (See [13]-[21], and the references therein.) However, in the context of resilient tracking control, there exist only a few studies in the literature. For example, in [3], the design of a specific deception attack, false data injection (FDI) attack, violating both feedback and forward channels, is considered in the sense that the attack can heavily destroy the tracking performance without being detected. The tracking problem of heterogeneous dynamical networks in the presence of malicious cyber attacks is investigated in [22]. The attacker therein launches asynchronous attacks on both controllers' and observers' communication channels. The proposed algorithm provides sufficient conditions on the length of attacking intervals in order to achieve the tracking objective. The distributed tracking control problem of multiagent systems under a strategic DoS attack, whose dynamics are modeled by a random Markov process, is addressed in [23]. Based on a hybrid stochastic secure control framework, a distributed resilient control law is developed to achieve exponential consensus tracking in a mean square sense. In [24], the tracking control of mobile robots in the presence of DoS attacks is studied. To ensure both the tracking convergence and efficient usage of communication recourses, a general hybrid model consisting of an event-triggering strategy and DoS attacks is established. Nevertheless, it should be pointed out that the existing literature concentrates primarily on one specific type of attack, and relatively few results deal with the tracking control problem in the presence of different attacks on communication channels. In some practical situations, it is quite common for a crafty adversary to launch assorted attacks of different models and strategies so that he or she could heavily disrupt the tracking performance. To the best of our knowledge, the resilient tracking control problem in NCSs subject to the presence of different cyber attacks that are modeled in a unified framework has not been adequately addressed and the problem remains challenging when the NCS is operated and controlled via some digital and unprotected communication networks, which motivates the current study.

In this article, we aim to achieve resilient tracking control for an NCS operating over a digital and unsafe communication network in the presence of simultaneous data quantization and two malicious attacks, including DoS attacks and deception attacks on the communication channels. Furthermore, we consider that the NCS also suffers from both UBB process noise and measurement noise. More specifically, two logarithmic quantizers will be considered over each of controllerto-plant (CP) and sensor-to-controller (SC) channels so as to adequately account for the quantization effect on the resilient tracking and estimation performance. The DoS attack will be launched by an adversary on the $\mathrm{CP}$ communication channel so as to prevent the physical plant from receiving the tracking control command. On the other hand, the deception attack will be considered on the SC communication channel so that the attacker can severely damage the tracking performance by modifying the measurement outputs meaningfully. By fully exploring two different attack models and strategies, the adversary aims to disrupt the tracking control performance of the system in a subtle way. The main contributions are summarized as follows.

- A resilient set-membership tracking control method is developed such that the true state of the system is guaranteed to be included in a bounding ellipsoidal set of the reference state in the simultaneous presence of UBB process and measurement noises as well as DoS attacks and deception attacks.

- A resilient set-membership estimation technique is delicately proposed on account of the scenario that the full states of the system may not be trustworthy and/or available at the controller side due to the existence of an adversary. The estimation procedure includes two steps: a) a predicted state estimate step and b) an updated state estimate step. The two-step estimation determines a group of confidence sets which contain the true state of the system while the attack may be present.

- A recursive convex optimization algorithm, which is based on a recursive computation of the reference state ellipsoid and confidence state estimation ellipsoids, is presented to determine the desired gain parameters for both the resilient tracking controller and estimator in an NCS whose $\mathrm{CP}$ and $\mathrm{SC}$ communication channels are under the presence of DoS and deception attacks, respectively.

The reminder of this paper is organized as follows. In Section II, models of DoS and deception attacks are presented and the system dynamics subject to these attacks and UBB noises are formulated. The observer-based tracking control protocol and the two-step state estimator are also constructed in this section. Main results are presented in Section III where resilient tracking control and estimation design criteria are derived. Section III also includes a recursive convex optimization algorithm through which the design parameters of interest are calculated. In Section IV, an internet-based threetank system (ITTS) is employed to illustrate the effectiveness of the proposed method. Finally, Section V draws a conclusion.

\section{Problem Formulation}

The purpose of this article is to design a resilient tracking control algorithm in the case that there exist DoS attacks on quantized control signals and deception attacks on quantized measurement outputs. The design criteria are presented in detail in Section III-A. To provide a solution for NCSs from a practical engineering perspective, it is anticipated that sensors, controllers, and actuators are at the distance from each other, i.e., mutually interconnected through a digital and unprotected communication network as shown in Fig. 1. In such a communication setting, it is expected that the transmitted signals are subject to quantization effects and are also exposed to cyber attacks. Details of data quantization and cyber attacks models are discussed in Section II-B and II-C, respectively.

\section{A. The System Model}

The physical plant is described by a discrete time-varying linear system of the following form:

$$
\left\{\begin{array}{l}
x_{k+1}=A_{k} x_{k}+B_{k} u_{k}+B_{k}^{w} w_{k} \\
y_{k}=C_{k} x_{k}+D_{k} v_{k}
\end{array}\right.
$$

where $x_{k} \in \mathbb{R}^{n_{x}}, u_{k} \in \mathbb{R}^{n_{u}}$, and $y_{k} \in \mathbb{R}^{n_{y}}$ are, respectively, the state vector, the control input, and the measurement output; 


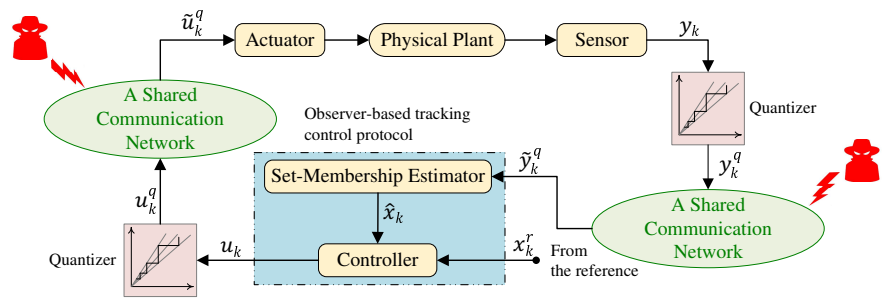

Fig. 1. Schematic of resilient observer-based tracking control over shared communication networks subject to cyber attacks.

$A_{k}, B_{k}, B_{k}^{w}, C_{k}$, and $D_{k}$ are time-varying matrices with appropriate dimensions; $x(0)=x_{0}$ is a given initial state of the system; $w_{k}$ and $v_{k}$ are the process noise and measurement noise, respectively, which are UBB. Before proceeding further, the following definitions and assumption are introduced.

Definition 1: [25] A nonlinearity $\varphi(\cdot): \mathbb{R}^{n} \mapsto \mathbb{R}^{n}$ is said to satisfy a sector condition if

$$
\left(\varphi(\varepsilon)-\Omega_{1} \varepsilon\right)^{T}\left(\varphi(\varepsilon)-\Omega_{2} \varepsilon\right) \leq 0, \forall \varepsilon \in \mathbb{R}^{n}
$$

for some real matrices $\Omega_{1}, \Omega_{2} \in \mathbb{R}^{n \times n}$, where $\Omega=\Omega_{2}-\Omega_{1}$ is a positive-definite symmetric matrix. In this case, the vectorvalued nonlinearity $\varphi(\cdot)$ belongs to the sector $\left[\Omega_{1}, \Omega_{2}\right]$.

Definition 2: [26] A bounded ellipsoid is denoted as $\mathscr{E}(c, P, n) \triangleq\left\{\zeta \in \mathbb{R}^{n}:(\zeta-c)^{T} P^{-1}(\zeta-c) \leq 1\right\}$, where $c \in \mathbb{R}^{n}$ is the center and $P>0$ is the shape matrix of the ellipsoid. Let $E \in \mathbb{R}^{n \times m}$ with $\operatorname{rank}(E)=m \leq n$ be a lower triangular matrix whose every diagonal element is positive. By a Cholesky factorization, we have $P=E E^{T}>0$. Hence, an alternative representation of the ellipsoid is $\mathscr{E} \triangleq\{\zeta: \zeta=$ $c+E z,\|z\| \leq 1\}$. The size of the ellipsoid is a function of the squared shape matrix $P$ which can be measured by means of trace $(P)$, that is, the sum of squared semiaxes lengths.

Assumption 1: The system noises $w_{k}$ and $v_{k}$ are confined to the following specified ellipsoidal sets:

$$
\begin{gathered}
\mathcal{W}_{k} \triangleq\left\{w_{k}: w_{k}^{T} W_{k}^{-1} w_{k} \leq 1\right\} \\
\mathcal{V}_{k} \triangleq\left\{v_{k}: v_{k}^{T} V_{k}^{-1} v_{k} \leq 1\right\}
\end{gathered}
$$

where $W_{k}=W_{k}^{T}>0$ and $V_{k}=V_{k}^{T}>0$ are time-varying matrices of compatible dimensions.

Remark 1: The UBB assumption of process and measurement noises is fully justified in the literature [27], [28] and therefore, it is omitted here.

\section{B. Data Quantization}

In a typical NCS, system components, such as sensors, controllers, and actuators, are remotely connected, and thus data transmissions among these components need to be carried out through some digital communication network medium. In this sense, the quantization effects and cyber attack impacts on both control signal and measurement output data are inevitable. It is our aim to investigate the problem of resilient observer-based tracking control for the system (1) via a digital and unprotected communication network. In particular, we consider that the signal is quantized by a logarithmic quantizer prior to enter the communication network, and then it may be compromised by a cyber attacker while being transmitted via communication network channels.

For the logarithmic quantizer, let an $m$-dimensional vector signal as $\epsilon_{k}=\left[\epsilon_{1, k}, \epsilon_{2, k}, \ldots, \epsilon_{m, k}\right]^{T} \in \mathbb{R}^{m}$. Then quantizer $q(\cdot)$ is expressed as

$$
\epsilon_{k}^{q}=q\left(\epsilon_{k}\right)=\left[q_{1}\left(\epsilon_{1, k}\right), q_{2}\left(\epsilon_{2, k}\right), \ldots, q_{m}\left(\epsilon_{m, k}\right)\right]^{T}
$$

where $\epsilon_{k}^{q} \in \mathbb{R}^{m}$ is the quantized signal. For each $q_{\ell}(\cdot) 1 \leq$ $\ell \leq m$, suppose that the set of quantization levels is described by

$$
\begin{aligned}
& \mho_{\ell}:=\left\{ \pm \mu_{\ell, i}: \mu_{\ell, i}=\rho_{\ell}^{i} \mu_{\ell, 0}, \quad i=0, \pm 1, \pm 2, \ldots\right\} \cup\{0\} \\
& 0<\rho_{\ell}<1, \mu_{\ell, 0}>0
\end{aligned}
$$

where $\rho_{\ell}$ is the quantization density and $\mu_{\ell, 0}$ is a scaling constant. Then from [29], the logarithmic quantizer $q_{\ell}(\cdot)$ is defined as

$$
q_{\ell}\left(\epsilon_{\ell, k}\right)= \begin{cases}\mu_{\ell, i}, & \frac{1+\rho_{\ell}}{2} \mu_{\ell, i} \leq \epsilon_{\ell, k} \leq \frac{1+\rho_{\ell}}{2 \rho_{\ell}} \mu_{\ell, i} \\ 0, & \epsilon_{\ell, k}=0 \\ -q_{\ell}\left(-\epsilon_{\ell, k}\right), & \epsilon_{\ell, k}<0 .\end{cases}
$$

A small $\rho_{\ell}$ implies coarse quantization and a large $\rho_{\ell}$ means dense quantization. Denoting $H_{1}=\operatorname{diag}_{m}\left\{2 \rho_{\ell} /\left(1+\rho_{\ell}\right)\right\}$ and $H_{2}=\operatorname{diag}_{m}\left\{2 /\left(1+\rho_{\ell}\right)\right\}$, it can be easily checked from the above definition (6) that the quantized signal $\epsilon_{k}^{q}$ satisfies the following inequality:

$$
\left(\epsilon_{k}^{q}-H_{1} \epsilon_{k}\right)^{T}\left(\epsilon_{k}^{q}-H_{2} \epsilon_{k}\right) \leq 0 .
$$

Since $0<\rho_{\ell}<1$, one can check that $0 \leq H_{1}<I \leq H_{2}$. Then $\epsilon_{k}^{q}$ can be decomposed as

$$
\epsilon_{k}^{q}=H_{1} \epsilon_{k}+\sigma\left(\epsilon_{k}\right)
$$

where $\sigma\left(\epsilon_{k}\right)$ is a nonlinear vector-valued function which, from Definition 1, satisfies

$$
\sigma\left(\epsilon_{k}\right)^{T}\left(\sigma\left(\epsilon_{k}\right)-H \epsilon_{k}\right) \leq 0
$$

with $H=H_{2}-H_{1}$.

Remark 2: The use of network in tracking control not only raises concern over security issues but also renders the assumption of data transmission with infinite precision no longer valid due to signal quantization or limited communication capacity. It is therefore of great significance to consider the quantization effects during the network transmission. A rich body of literature in quantization of sensor measurement and/or control input signals is available, among which two quantization approaches are widely employed: 1) linear (or uniform) quantization and 2) logarithmic quantization. As discussed in [29]-[31], the logarithmic quantization, whose step size increases exponentially with respect to the increase in the input, outperforms the linear quantization in control problems. This is due to the fact that, in the logarithmic quantization, the relative quantization error is guaranteed to be roughly constant, which serves as an important feature in many applications.

Remark 3: Since the quantization effects are considered on both the control signal $u_{k}$ and the measurement output $y_{k}$, the corresponding matrices, scalars, and nonlinear vector-valued function, i.e., $H_{1}, H_{2}, H, \rho_{\ell}, \mu_{\ell, 0}$, and $\sigma(\cdot)$ will be denoted with superscripts $u$ and $y$, respectively, in the reminder of this article.

\section{Cyber Attacks Models}

Consider the scenario that there exists an attacker who deteriorates the remote estimation performance by injecting certain deception signals into the true value of the measurement output $y_{k}$ and also degrades the remote tracking performance through jamming the wireless communication channels between the controller and the physical plant, as shown in Fig. 1. 
Before introducing the cyber attacks models, there are some assumptions on the system knowledge being available to the attacker for launching a successful attack. Regarding the DoS attack on control signals, the attacker is an active adversary in the sense that the control signal will be dropped once the wireless channels are successfully jammed. After the occurrence of the DoS attack, one of the following cases occurs: 1) the control signal will be successfully received by the physical plant if the attacker completely fails to jam the corresponding communication channels. This case might occur if the attacker has to desist from jamming certain channels due to a limited energy budget; 2) the control signal will be partially received if the communication channels are not heavily jammed; and 3) the control signal will be completely lost if the communication channels are under a severe DoS attack. For the deception attack on measurement outputs, in the first phase, the attacker must have this ability to know the accurate value of the measurement output in real time, and in the second phase, the attacker must be able to modify the true value of the measurement output to his or her arbitrary one.

Motivated by the above observation, the actual control input, which is under a DoS attack and received by the physical plant, is proposed as

$$
\tilde{u}_{k}=\Gamma_{k} u_{k}
$$

where $\Gamma_{k} \in \mathbb{R}^{n_{u} \times n_{u}}$ represents an attack model parameter matrix prescribed by the attacker and it has the following diagonal structure:

$$
\left\{\begin{array}{l}
\Gamma_{k}=\operatorname{diag}\left\{\gamma_{1, k}, \gamma_{2, k}, \ldots, \gamma_{n_{u}, k}\right\} \\
\underline{\gamma}_{j, k} \leq \gamma_{j, k} \leq \bar{\gamma}_{j, k}, j=1,2, \ldots, n_{u}
\end{array}\right.
$$

where $\underline{\gamma}_{j, k}, \bar{\gamma}_{j, k} \in[0,1]$ are known scalars representing the lower and upper bounds of the unknown parameter $\gamma_{j, k}$.

Remark 4: The unknown parameter $\gamma_{j, k}$ represents the transmission status of the $j$ th control signal's component so as to reflect the jamming status of the communication channel under attack. If $\gamma_{j, k}=1$, there is no DoS attack on the $j$ th control signal's component at time $k$ and therefore, this component can be successfully delivered to the physical plant. When $0<\gamma_{j, k}<1$, it represents the case of partial transmission of the $j$ th component. The case $\gamma_{j, k}=0$ characterizes the worst case scenario on transmission of the $j$ th control signal's component, which means the $j$ th component is completely lost while being transmitted at time $k$.

To facilitate subsequent analysis, we define the following matrices

$$
\begin{aligned}
& \hat{\Gamma}_{k}=\operatorname{diag}\left\{\frac{\bar{\gamma}_{1, k}+\underline{\gamma}_{1, k}}{2}, \frac{\bar{\gamma}_{2, k}+\underline{\gamma}_{2, k}}{2}, \ldots, \frac{\bar{\gamma}_{n_{u}, k}+\underline{\gamma}_{n_{u}, k}}{2}\right\} \\
& \check{\Gamma}_{k}=\operatorname{diag}\left\{\frac{\bar{\gamma}_{1, k}-\underline{\gamma}_{1, k}}{2}, \frac{\bar{\gamma}_{2, k}-\underline{\gamma}_{2, k}}{2}, \ldots, \frac{\bar{\gamma}_{n_{u}, k}-\underline{\gamma}_{n_{u}, k}}{2}\right\} .
\end{aligned}
$$

Then, one has

$$
\Gamma_{k}=\hat{\Gamma}_{k}+\tilde{\Gamma}_{k}
$$

where $\tilde{\Gamma}_{k}=\operatorname{diag}\left\{\tilde{\gamma}_{1, k}, \tilde{\gamma}_{2, k}, \ldots, \tilde{\gamma}_{n_{u}, k}\right\}$ and $\left|\tilde{\gamma}_{j, k}\right| \leq\left(\bar{\gamma}_{j, k}-\right.$ $\left.\underline{\gamma}_{j, k}\right) / 2$. Thus,

$$
\left\|\tilde{\Gamma}_{k}\right\| \leq \check{\Gamma}_{k} .
$$

Furthermore, the signal generated by the attacker for the deception attack is proposed as

$$
y_{k}^{a}=-y_{k}+\delta_{k}
$$

where $\delta_{k}$ is a UBB signal belonging to the following ellipsoid

$$
\mathcal{S}_{k} \triangleq\left\{\delta_{k}: \delta_{k}^{T} S_{k}^{-1} \delta_{k} \leq 1\right\}
$$

with $S_{k}=S_{k}^{T}>0$

Remark 5: Note that the signal $\delta_{k}$ used by the attacker to launch the deception attack has similar form with the system noises $w_{k}$ and $v_{k}$. As discussed in [32], considering the signal $\delta_{k}$ to be unknown but confined to a specified ellipsoidal set not only makes the deception attack difficult to be distinguished by the detectors but also facilitates the attacker to successfully bypass the most widely employed false data detector, namely, the $\chi^{2}$ detector since this detector is only effective when system noises comply with Gaussian distribution.

The actual measurement output, which is under a deception attack and sent to the estimator, can be represented by

$$
\tilde{y}_{k}=y_{k}+\Lambda_{k} y_{k}^{a}
$$

where the matrix $\Lambda_{k}$ represents the physical constraints imposed on the attack signal due to some hardware constraints including device saturations, bandwidth limitations, and channel fading. This matrix is assumed to have the following diagonal structure

$$
\left\{\begin{array}{l}
\Lambda_{k}=\operatorname{diag}\left\{\lambda_{1, k}, \lambda_{2, k}, \ldots, \lambda_{n_{y}, k}\right\} \\
\underline{\lambda}_{s, k} \leq \lambda_{s, k} \leq \bar{\lambda}_{s, k}, \quad s=1,2, \ldots, n_{y}
\end{array}\right.
$$

where $0 \leq \underline{\lambda}_{s, k}<1, \bar{\lambda}_{s, k} \geq 1$ are known scalars describing the lower and upper bounds on $\lambda_{s, k}$.

Remark 6: The parameter $\lambda_{s, k}$ describes how the physical constraints affect the behavior of the deception attack. If $\lambda_{s, k}=1$, there exists a complete deception attack on the $s$ th component of the measurement output as the attacker plans. When $0 \leq \lambda_{s, k}<1$ or $\lambda_{s, k}>1$, the deception signal $y_{k}^{a}$ might be degraded or amplified, respectively, from the attacker's point of view. As a result, taking physical constraints into consideration offers a comprehensive yet realistic deception attack model which reflects the impacts of hardware constraints that the attacker would be confronted with.

By denoting $\underline{\Lambda}_{k}=\operatorname{diag}\left\{\underline{\lambda}_{1, k}, \underline{\lambda}_{2, k}, \ldots, \underline{\lambda}_{n_{y}, k}\right\}$ and $\bar{\Lambda}_{k}=$ $\operatorname{diag}\left\{\bar{\lambda}_{1, k}, \bar{\lambda}_{2, k}, \ldots, \bar{\lambda}_{n_{y}, k}\right\}$, the lower- and upper-bounds on $\lambda_{p, k},(18)$, can be written in a compact form

$$
\underline{\Lambda}_{k} \leq \Lambda_{k} \leq \bar{\Lambda}_{k}
$$

To simplify the subsequent derivation, the deception attack signal $\Lambda_{k} y_{k}^{a}$ can be split into two terms as

$$
\Lambda_{k} y_{k}^{a}=\underline{\Lambda}_{k} y_{k}^{a}+\psi\left(y_{k}^{a}\right)
$$

where $\psi\left(y_{k}^{a}\right)$ is a nonlinear vector-valued function which from Definition 1 satisfies a sector condition with $\Omega_{1}=0$ and $\Omega_{2}=$ $\tilde{\Lambda}_{k}$, in which $\tilde{\Lambda}_{k}=\bar{\Lambda}_{k}-\underline{\Lambda}_{k}>0$, i.e., $\psi\left(y_{k}^{a}\right)$ satisfies the following inequality:

$$
\psi^{T}\left(y_{k}^{a}\right)\left(\psi\left(y_{k}^{a}\right)-\tilde{\Lambda}_{k} y_{k}^{a}\right) \leq 1 .
$$

\section{The Resilient Observer-Based Tracking Control Protocol}

On account of DoS and deception attacks, and quantization effects discussed above, the original physical plant (1) can be reformulated as

$$
\left\{\begin{array}{l}
x_{k+1}=A_{k} x_{k}+B_{k} \tilde{u}_{k}^{q}+B_{k}^{w} w_{k} \\
y_{k}=C_{k} x_{k}+D_{k} v_{k} \\
\tilde{u}_{k}^{q}=\Gamma_{k} u_{k}^{q}, \quad u_{k}^{q}=H_{1}^{u} u_{k}+\sigma^{u}\left(u_{k}\right) \\
\tilde{y}_{k}^{q}=y_{k}^{q}+\Lambda_{k} y_{k}^{q, a}, \quad y_{k}^{q}=H_{1}^{y} y_{k}+\sigma^{y}\left(y_{k}\right) \\
y_{k}^{q, a}=-y_{k}^{q}+\delta_{k} .
\end{array}\right.
$$


In engineering practice, sensing and computing capabilities of a sensor are often restricted to a small voltage source, and thus its performance can be degraded by some potential attacks and external disturbances. Thus, the assumption of full state information availability and/or integrity may not be realistic. In what follows, a two-step estimator is developed to monitor and observe the system's true state as

$$
\left\{\begin{array}{l}
\text { Predicted (a priori) state estimate: } \\
\hat{x}_{k+1 \mid k}=\hat{A}_{k} \hat{x}_{k} \\
\text { Updated (a posteriori) state estimate: } \\
\hat{x}_{k+1}=\hat{x}_{k+1 \mid k}+\hat{B}_{k+1}\left(\tilde{y}_{k+1}^{q}-C_{k+1} \hat{x}_{k+1 \mid k}\right)
\end{array}\right.
$$

where $\hat{x}_{k}$ is the estimation of the state $x_{k} ; \hat{A}_{k}$ and $\hat{B}_{k+1}$ are time-varying parameter matrices of the estimator to be designed, and the initial condition of the estimator is $\hat{x}_{0}$.

Hence, the following observer-based tracking control protocol, which utilizes the estimated state instead of the full state information measured by sensors, is proposed

$$
u_{k}=M_{k}\left(\hat{x}_{k}-x_{k}^{r}\right)
$$

where $M_{k}$ is the controller gain to be designed; and $x_{k}^{r}$ is the state of the specified reference model whose dynamics are given by

$$
x_{k+1}^{r}=A_{k}^{r} x_{k}^{r}, x^{r}(0)=x_{0}^{r} .
$$

\section{E. The Resilient Set-Membership Tracking Control Problem}

We next develop a suitable set-membership tracking control framework through which the true state of the system will be enclosed in some confidence region of the reference state and also, simultaneously, some confidence region of the system's state estimation despite the existence of UBB noises and cyber attacks. To proceed with, the definition of the two-step setmembership state estimation is firstly presented.

Definition 3: System (22) is said to achieve set-membership state estimation at the predicated (a priori) state estimate step under the tracking protocol (24) if there exist gain sequences $\hat{A}_{k}$ and $M_{k}$ such that the system's state $x_{k+1}$ resides in a state prediction ellipsoid $\mathcal{X}_{k+1 \mid k}$, which always contains the true state of the system, where

$$
\begin{aligned}
\mathcal{X}_{k+1 \mid k} \triangleq\left\{x_{k+1}:\right. & \left(x_{k+1}-\hat{x}_{k+1 \mid k}\right)^{T} \\
& \left.\times P_{k+1 \mid k}^{-1}\left(x_{k+1}-\hat{x}_{k+1 \mid k}\right) \leq 1\right\}
\end{aligned}
$$

for all $k \in \mathbb{N}$; UBB process noise $w_{k} \in \mathcal{W}_{k}$ and UBB measurement noise $v_{k} \in \mathcal{V}_{k}$ with $P_{k+1 \mid k}=P_{k+1 \mid k}^{T}>0$ representing a time-varying matrix.

Definition 4: System (22) is said to achieve set-membership state estimation at the updated (a posteriori) state estimate step under the tracking protocol (24) if there exists a gain sequence $\hat{B}_{k+1}$ such that the system's state $x_{k+1}$ resides in a state estimation ellipsoid $\mathcal{X}_{k+1}$, which always contains the true state of the system, where

$$
\mathcal{X}_{k+1} \triangleq\left\{x_{k+1}:\left(x_{k+1}-\hat{x}_{k+1}\right)^{T} P_{k+1}^{-1}\left(x_{k+1}-\hat{x}_{k+1}\right) \leq 1\right\}
$$

for all $k \in \mathbb{N}$; UBB process noise $w_{k} \in \mathcal{W}_{k}$ and UBB measurement noise $v_{k} \in \mathcal{V}_{k}$ with $P_{k+1}=P_{k+1}^{T}>0$ representing a time-varying matrix.

Remark 7: Resilient estimation has been deemed as an effective means of guaranteeing a reliable estimation of the system's state in the simultaneous presence of noises and attacks, and thus has been intensively studied in the field of NCSs. Arguably, prior work on resilient estimation in the presence of noises and attacks can be divided into two categories depending on the noise model [33]: i) Stochastic noises (e.g., Guassian noise) [34]-[37] and ii) bounded nonstochastic noises (e.g., $l_{2}$-norm energy-bounded noise) [38][41]. Although fruitful results have been available on resilient estimation, it should be pointed out that modeling the noise in a stochastic framework usually requires accurate statistical properties of the noise such as known mean and covariance, which is conservative for some practical applications when the noise is unknown. In an energy-bounded noise model, e.g., in the $H_{\infty}$ sense, it is assumed that the noise has norm-bounded energy so as to satisfy the aim of finding the worst-case solution to the estimation problem. Furthermore, the objective of a conventional estimation problem is to compute a single vector estimation $\hat{x}_{k}$ with regard to the system's state such that $\lim _{k \rightarrow \infty}\left\|x_{k}-\hat{x}_{k}\right\|=0$ in the asymptotic convergence case. However, such an estimation approach only provides a pointwise estimation of the system's state [28]. Therefore, there is no guarantee that the distribution of the system's state can be achieved within a confidence region where all true states of the system may reside in, specially when there exist unpredictable environmental changes due to the influences of cyber attacks and noises. As a result, an alternative method called setmembership estimation is developed in the literature [42]. The core idea of this method is to calculate a bounding ellipsoidal set in state-space, which always encloses the true state of the system by assuming UBB noise signals [43], [44]. Indeed, an assumption of UBB noise eliminates the requirement of prior knowledge of the accurate statistical characteristics of noise because only the knowledge of a bound on the realization is needed. During the past two decades, the set-membership method has been intensively studied for different problem formulations of various system models [27], [28], [45]-[47]. However, to the best of the authors' knowledge, there are rare results available that consider the conjunct problem of resilient tracking control and set-membership estimation over a shared communication network subject to two different cyber attacks independently corrupting the sensor data and control signals, and UBB process and measurement noises.

We then present the definition of the proposed resilient setmembership tracking control.

Definition 5: System (22) subject to DoS and deception attacks on control signals and measurement outputs is said to achieve resilient set-membership tracking control w.r.t. the reference model (25) under the tracking protocol (24) if there exist gain sequences $\hat{A}_{k}$ and $M_{k}$ such that the system's state $x_{k+1}$ resides in a reference state ellipsoid $\mathcal{T}_{k+1}$, which always contains the true state of the system, where

$$
\mathcal{T}_{k+1} \triangleq\left\{x_{k+1}:\left(x_{k+1}-x_{k+1}^{r}\right)^{T} R_{k+1}^{-1}\left(x_{k+1}-x_{k+1}^{r}\right)\right\} \leq 1
$$

for all $k \in \mathbb{N}$; UBB process noise $w_{k} \in \mathcal{W}_{k}$ and UBB measurement noise $v_{k} \in \mathcal{V}_{k}$ with $R_{k+1}=R_{k+1}^{T}>0$ representing a time-varying matrix.

The initial state $x_{0}$ satisfies the following assumption.

Assumption 2: The initial state of the system belongs to 
given ellipsoids

$$
\begin{aligned}
& \mathcal{T}_{0} \triangleq\left\{x_{0}:\left(x_{0}-x_{0}^{r}\right)^{T} R_{0}^{-1}\left(x_{0}-x_{0}^{r}\right) \leq 1\right\} \\
& \mathcal{X}_{0} \triangleq\left\{x_{0}:\left(x_{0}-\hat{x}_{0}\right)^{T} P_{0}^{-1}\left(x_{0}-\hat{x}_{0}\right) \leq 1\right\} .
\end{aligned}
$$

From Definitions 3, 4, and 5, resilient set-membership tracking control and estimation problem to be addressed is now stated as: For prescribed time-varying matrices $\Gamma_{k}, \Lambda_{k}$, scalars $\rho_{\ell}^{u}, \rho_{\ell}^{y}, \mu_{\ell, 0}^{u}, \mu_{\ell, 0}^{y}$ satisfying (5), UBB deception attack $\delta_{k} \in \mathcal{S}_{k}$, UBB noises $w_{k} \in \mathcal{W}_{k}$ and $v_{k} \in \mathcal{V}_{k}$, the objective is to design a desired tracking control protocol in the form of (24) and to find appropriate matrix sequences $R_{k+1}, P_{k+1 \mid k}$, and $P_{k+1}$ such that the system's one-step ahead state $x_{k+1}$ always resides in the ellipsoids $\mathcal{X}_{k+1 \mid k}, \mathcal{X}_{k+1}$, and $\mathcal{T}_{k+1}$, simultaneously.

\section{MAIN RESUlts}

\section{A. Design Criteria}

In the following text, we first present two theorems, which establish sufficient conditions on the existence of a two-step set-membership estimator (23) and a desired tracking control protocol (24) such that (26), (27), and (28) hold.

Theorem 1: For the system (22) subject to UBB noises $w_{k} \in \mathcal{W}_{k}$ and $v_{k} \in \mathcal{V}_{k}$, and DoS attacks and deception attacks on control signals and measurement outputs, respectively, suppose that at time $k$ the system's state $x_{k}$ belongs to its state estimation ellipsoid $\left(x_{k}-\hat{x}_{k}\right)^{T} P_{k}^{-1}\left(x_{k}-\hat{x}_{k}\right) \leq 1$ and its reference state ellipsoid $\left.\left(x_{k}-x_{k}^{r}\right)^{T} R_{k}^{-1}\left(x_{k}-x_{k}^{r}\right)\right\} \leq 1$. Then, for any prescribed time-varying matrices $\Gamma_{k}, \Lambda_{k}$, scalars $\rho_{\ell}^{u}, \rho_{\ell}^{y}, \mu_{\ell, 0}^{u}, \mu_{\ell, 0}^{y}$ satisfying (5), and UBB deception attack $\delta_{k} \in \mathcal{S}_{k}$, the system's one-step ahead state $x_{k+1}$ always resides in the state prediction ellipsoid $\mathcal{X}_{k+1 \mid k}$ and the reference state ellipsoid $\mathcal{T}_{k+1}$, if there exist matrix sequences $P_{k+1 \mid k}>0$, $R_{k+1}>0, \hat{A}_{k}, M_{k}$, and scalar sequences $\tau_{m, k}>0$, $m=1,2, \ldots, 4$, such that

$$
\left[\begin{array}{ccc}
-I & 0 & \Phi_{k} \\
* & -P_{k+1 \mid k} & \Pi_{k}^{p} \\
* & * & \Theta_{k}^{p}
\end{array}\right] \leq 0, \quad\left[\begin{array}{ccc}
-I & 0 & \Phi_{k} \\
* & -R_{k+1} & \Pi_{k}^{c} \\
* & * & \Theta_{k}^{c}
\end{array}\right] \leq 0
$$

for all $k \in \mathbb{N}$, where

$$
\begin{aligned}
& \Pi_{k}^{p}=\left[\begin{array}{lllll}
\Pi_{11}^{p} & A_{k} E_{k} & B_{k}^{w} & B_{k} & B_{k} \hat{\Gamma}_{k}
\end{array}\right] \\
& \Pi_{11}^{p}=\left(A_{k}-\hat{A}_{k}+B_{k} \hat{\Gamma}_{k} H_{1}^{u} M_{k}\right) \hat{x}_{k}-B_{k} \hat{\Gamma}_{k} H_{1}^{u} M_{k} x_{k}^{r} \\
& \Pi_{k}^{c}=\left[\begin{array}{lllll}
\Pi_{11}^{c} & A_{k} L_{k} & B_{k}^{w} & B_{k} & B_{k} \hat{\Gamma}_{k}
\end{array}\right] \\
& \Pi_{11}^{c}=\left(A_{k}-A_{k}^{r}-B_{k} \hat{\Gamma}_{k} H_{1}^{u} M_{k}\right) x_{k}^{r}+B_{k} \hat{\Gamma}_{k} H_{1}^{u} M_{k} \hat{x}_{k} \\
& \Phi_{k}=\left[\check{\Gamma}_{k} H_{1}^{u} M_{k}\left(\hat{x}_{k}-x_{k}^{r}\right) \quad 0 \quad 0000\right]
\end{aligned}
$$

and $\Theta_{k}^{p}=\left[\Theta_{\alpha, \beta}^{c}(k)\right]_{5 \times 5}$ and $\Theta_{k}^{c}=\left[\Theta_{\alpha, \beta}^{c}(k)\right]_{5 \times 5}$ are timevarying sparse symmetric block matrices with their nonzero entries given by

$$
\begin{aligned}
& \Theta_{1,1}^{p}(k)=-1+\tau_{3, k}+\tau_{4, k}, \Theta_{1,1}^{c}(k)=-1+\tau_{1, k}+\tau_{2, k} \\
& \Theta_{1,5}^{p}(k)=\Theta_{1,5}^{c}(k), \Theta_{1,5}^{c}(k)=(1 / 2)\left(\hat{x}-x_{k}^{r}\right)^{T} M_{k}^{T} H^{u} \\
& \Theta_{2,2}^{p}(k)=-\tau_{3, k} I, \Theta_{2,2}^{c}(k)=-\tau_{1, k} I \\
& \Theta_{3,3}^{p}(k)=-\tau_{4, k} W_{k}^{-1}, \Theta_{3,3}^{c}(k)=-\tau_{2, k} W_{k}^{-1} \\
& \Theta_{4,4}^{p}(k)=\Theta_{4,4}^{c}(k)=-I \\
& \Theta_{5,5}^{p}(k)=\Theta_{5,5}^{c}(k)=2 \check{\Gamma}_{k}^{2}-I .
\end{aligned}
$$

Proof: See the Appendix.

Theorem 2: For the system (22) subject to UBB noises $w_{k} \in$ $\mathcal{W}_{k}$ and $v_{k} \in \mathcal{V}_{k}$, and DoS attacks and deception attacks on control signals and measurement outputs, respectively, suppose that the one-step ahead state $x_{k+1}$ belongs to its state prediction ellipsoid $\left.\left(x_{k+1}-\hat{x}_{k+1 \mid k}\right)^{T} P_{k+1 \mid k}^{-1}\left(x_{k+1}-\hat{x}_{k+1 \mid k}\right)\right\} \leq 1$. Then, for any prescribed time-varying matrices $\Gamma_{k}, \Lambda_{k}$, scalars $\rho_{\ell}^{u}, \rho_{\ell}^{y}, \mu_{\ell, 0}^{u}, \mu_{\ell, 0}^{y}$ satisfying (5), and UBB deception attack $\delta_{k} \in \mathcal{S}_{k}$, such a state belongs to its state estimation ellipsoid $\mathcal{X}_{k+1}$, if there exist matrix sequences $P_{k+1}>0, \hat{B}_{k+1}$, $\tilde{S}_{k+1}>0$, and scalar sequences $\tau_{m, k}>0, m=5,6, \ldots, 8$, such that

$$
\left[\begin{array}{cc}
-P_{k+1} & \Pi_{k}^{e} \\
* & \Theta_{k}^{e}
\end{array}\right] \leq 0
$$

for all $k \in \mathbb{N}$, where

$$
\begin{aligned}
& \Pi_{k}^{e}=\left[\begin{array}{llllll}
\Pi_{11}^{e} & \Pi_{12}^{e} & \Pi_{13}^{e} & \Pi_{14}^{e} & \Pi_{15}^{e} & -\hat{B}_{k+1}
\end{array}\right] \\
& \Pi_{11}^{e}=-\hat{B}_{k+1}\left(\left(I-\underline{\Lambda}_{k+1}\right) H_{1}^{y}-I\right) C_{k+1} \hat{x}_{k+1 \mid k} \\
& \Pi_{12}^{e}=\left(I-\hat{B}_{k+1}\left(I-\underline{\Lambda}_{k+1}\right) H_{1}^{y} C_{k+1}\right) E_{k+1 \mid k} \\
& \Pi_{13}^{e}=-\hat{B}_{k+1}\left(I-\underline{\Lambda}_{k+1}\right) H_{1}^{y} D_{k+1} \\
& \Pi_{14}^{e}=-\hat{B}_{k+1} \underline{\Lambda}_{k+1}, \quad \Pi_{15}^{e}=-\hat{B}_{k+1}\left(I-\underline{\Lambda}_{k+1}\right)
\end{aligned}
$$

and $\Theta_{k}^{e}=\left[\Theta_{\alpha, \beta}^{e}(k)\right]_{6 \times 6}$ is a time-varying sparse symmetric block matrix with its nonzero entries given by

$$
\begin{aligned}
& \Theta_{1,1}^{e}(k)=-1+\tau_{5, k}+\tau_{6, k}+\tau_{7, k} \\
& \Theta_{1,5}^{e}(k)=(1 / 2) \tau_{8, k} \hat{x}_{k+1 \mid k}^{T} C_{k+1}^{T} H^{y} \\
& \Theta_{1,6}^{e}(k)=-(1 / 2) \tau_{8, k} \hat{x}_{k+1 \mid k}^{T} C_{k+1}^{T} H_{1}^{y} \tilde{\Lambda}_{k+1} \\
& \Theta_{2,2}^{e}(k)=-\tau_{5, k} I, \Theta_{2,5}^{e}(k)=(1 / 2) \tau_{8, k} E_{k+1 \mid k}^{T} C_{k+1}^{T} H^{y} \\
& \Theta_{2,6}^{e}(k)=-(1 / 2) \tau_{8, k} E_{k+1 \mid k}^{T} C_{k+1}^{T} H_{1}^{y} \tilde{\Lambda}_{k+1} \\
& \Theta_{3,3}^{e}(k)=-\tau_{6, k} V_{k+1}^{-1}, \Theta_{3,5}^{e}(k)=(1 / 2) \tau_{8, k} D_{k+1}^{T} H^{y} \\
& \Theta_{3,6}^{e}(k)=-(1 / 2) \tau_{8, k} D_{k+1}^{T} H_{1}^{y} \tilde{\Lambda}_{k+1} \\
& \Theta_{4,4}^{e}(k)=-\tilde{S}_{k+1}, \Theta_{4,6}^{e}(k)=(1 / 2) \tau_{8, k} \tilde{\Lambda}_{k+1} \\
& \Theta_{5,5}^{e}(k)=\Theta_{6,6}^{e}(k)=-\tau_{8, k} I, \Theta_{5,6}^{e}(k)=-\Theta_{4,6}^{e}(k)
\end{aligned}
$$

where the parameter $S_{k+1}$ can be calculated by $S_{k+1}=$ $\tau_{7, k} \tilde{S}_{k+1}^{-1}$.

Proof: See the Appendix.

Remark 8: By Theorems 1 and 2, the proposed resilient set-membership tracking control and two-step set-membership estimation problem can be transferred into the feasibility problem of a set of recursive linear matrix inequalities (RLMIs) (31) and (32). Thus, Theorems 1 and 2 provide criteria for designing tracking control and two-step estimation gain matrix sequences $M_{k}, \hat{A}_{k}$, and $\hat{B}_{k+1}$ such that the true state of the system is always included in the ellipsoids $\mathcal{T}_{k+1}, \mathcal{X}_{k+1 \mid k}$, and $\mathcal{X}_{k+1}$ despite the existence of UBB process noise, UBB measurement noise, DoS attacks on quantized control signals, and UBB deception attacks on quantized measurement outputs.

Remark 9: Notice that the developed resilient tracking control framework assumes only DoS attacks on the CP communication channels and deception attacks on the SC communication channels, respectively. The reasons are twofold. First, we consider that the attacker is a cunning adversary who launches cyber attacks under two different attack models and strategies for comprehensively compromising the sensor measurements and control signals. It has been well acknowledged that different attack strategies are generally stealthy to any defender. Second, in practice, some transmission layers between system components such as the CP communication channel may gain more protection than the others such as 
the SC communication channel. This is due to the fact that more security requires heavy weight computations and large memory capacity. However, the widely used cryptographic techniques to encrypt data in the SC communication channel are not sufficient due to the constraints of memory and weak processing capability of sensors [48]. Thus, from the attacker's perspective, it is much easier to launch a DoS attack on the $\mathrm{CP}$ channel because a DoS attack requires less system model knowledge and disruption resources to cause destruction.

\section{B. The Recursive Convex Optimization Algorithm}

In light of Theorems 1 and 2, the system's one-step ahead state is always confined in the reference state ellipsoid $\mathcal{T}_{k+1}$, its state prediction ellipsoid $\mathcal{X}_{k+1 \mid k}$, and its state estimation ellipsoid $\mathcal{X}_{k+1}$ if (31) and (32) hold. Thus, there exist three vectors $\vartheta_{z}, z=1,2,3$, satisfying $\left\|\vartheta_{z}\right\| \leq 1$ such that $x_{k+1}=x_{k}^{r}+L_{k+1} \vartheta_{1}, x_{k+1}=\hat{x}_{k+1}+E_{k+1} \vartheta_{2}$, and $x_{k+1}=\hat{x}_{k+1 \mid k}+E_{k+1 \mid k} \vartheta_{3}$, respectively. Furthermore, the centers of ellipsoids $\mathcal{X}_{k+1 \mid k}, \mathcal{X}_{k+1}$, and $\mathcal{T}_{k+1}$ are determined by (23) and (25), respectively. Note that even though Theorems 1 and 2 outline the principles of determining reference state ellipsoid, prediction state and estimation state ellipsoids, they do not provide an optimal reference state ellipsoid or optimal prediction state and estimation state ellipsoids. Hence, by applying a convex optimization approach, the proposed resilient set-membership tracking control and two-step set-membership estimation problems are cast into the following optimization problems (OPs) so as to find some optimal ellipsoids:

$$
\underset{R_{k+1}, P_{k+1 \mid k}, \hat{A}_{k}, M_{k}, \tau_{1, k}, \tau_{2, k}, \tau_{3, k}, \tau_{4, k}}{\operatorname{minimize}} \operatorname{Tr}\left(\Upsilon_{k+1}\right)
$$

subject to (31),

where $\Upsilon_{k+1}=\operatorname{diag}\left\{R_{k+1}, P_{k+1 \mid k}\right\}$, and

$$
\begin{array}{ll}
\underset{P_{k+1}, \hat{B}_{k+1}, \tilde{S}_{k+1}, \tau_{5, k}, \tau_{6, k}, \tau_{7, k}, \tau_{8, k}}{\operatorname{minimize}} & \operatorname{Tr}\left(P_{k+1}\right) \\
\text { subject to (32). } &
\end{array}
$$

Remark 10: Note that (31) and (32) are linear to $R_{k+1}$, $P_{k+1 \mid k}, P_{k+1}, \tilde{S}_{k+1}, \hat{A}_{k}, \hat{B}_{k+1}, M_{k}$, and $\tau_{m, k}, m=$ $1,2, \ldots, 8$. Thus, the OPs (33) and (34) can be solved by some existing semidefinite programming via an interior-point algorithm at each time step [49]. In (33) and (34), it can be observed that the traces of $\Upsilon_{k+1}$ and $P_{k+1}$ are minimized at each time step in an effort to find the smallest ellipsoids for the reference state, state predictions, and state estimations.

Based on the OPs (33) and (34), we are in a position to present a recursive algorithm which computes the gain matrix sequences for the observer-based tracking protocol (24) and also solves out the gain matrix sequences for the two-step set-membership estimator (23) as well as determining matrix sequences $R_{k+1}, P_{k+1 \mid k}, P_{k+1}$ for optimal ellipsoids.

\section{Recursive Convex Optimization Algorithm}

Step 1. Given initial conditions $x_{0}, \hat{x}_{0}, x_{0}^{r}$, known lower- and upper-bounds on $\gamma_{j, k}$ and $\lambda_{s, k}$ such that $\underline{\gamma}_{j, k}, \bar{\gamma}_{j, k} \in$ $[0,1], \underline{\lambda}_{s, k} \in[0,1), \bar{\lambda}_{s, k} \geq 1$, and given quantization parameters $\rho_{\ell}^{u}, \rho_{\ell}^{y}, \mu_{0}^{u}$, and $\mu_{0}^{y}$ satisfying (5). Choose suitable $W_{k}, V_{k}, R_{0}$ and $P_{0}$ such that (3), (4), (29) and (30) hold. Calculate $E_{0}$ and $L_{0}$ according to $P_{0}=$ $E_{0} E_{0}^{T}$ and $R_{0}=L_{0} L_{0}^{T}$. Set $k=0, x_{k}=x_{0}, \hat{x}_{k}=\hat{x}_{0}$, $x_{k}^{r}=x_{0}^{r}, E_{k}=E_{0}, L_{k}=L_{0}$. Let the simulation run recursively for $T_{n}$ seconds.
Step 2. Solve the OP (33) to obtain $R_{k+1}, P_{k+1 \mid k}, \hat{A}_{k}$, and $M_{k}$. Compute $L_{k+1}$ and $E_{k+1 \mid k}$ such that $R_{k+1}=$ $L_{k+1} L_{k+1}^{T}$ and $P_{k+1 \mid k}=E_{k+1 \mid k} E_{k+1 \mid k}^{T}$.

Step 3. Calculate the state prediction $\hat{x}_{k+1 \mid k}$ by (23).

Step 4. Solve the OP (34) to determine $P_{k+1}$, and $\hat{B}_{k+1}$. Obtain $E_{k+1}$ such that $P_{k+1}=E_{k+1} E_{k+1}^{T}$.

Step 5. Compute the reference state $x_{k+1}^{r}$ by (25) and the state estimation $\hat{x}_{k+1}$ by (23).

Step 6. If $k=T_{n}$ go to Step 7; otherwise, set $k=k+1$, go to Step 2.

Step 7. Output matrix sequences $\left\{R_{k+1}\right\},\left\{P_{k+1 \mid k}\right\},\left\{P_{k+1}\right\}$, $\left\{\hat{A}_{k}\right\},\left\{\hat{B}_{k+1}\right\},\left\{M_{k}\right\}$, the reference state ellipsoidal set $\mathcal{T}_{k+1}$, the state prediction ellipsoidal set $\mathcal{X}_{k+1 \mid k}$, and the state estimation ellipsoidal set $\mathcal{X}_{k+1}$. Exit.

\section{An Illustrative EXAMPLE}

In this section, the developed resilient observer-based tracking control method is applied to an internet-based three-tank system (ITTS) which is adopted from [50] as a benchmark system in order to investigate the resiliency of such a system subject to DoS and UBB deception attacks.

We first introduce the ITTS in which the physical plant, i.e., a three-tank system (TTS), is linked with the remote observerbased tracking controller and its two-step set-membership estimator through a shared communication network, as shown in Fig. 2. The three tanks in this system have been labeled as $T_{1}, T_{3}$, and $T_{2}$, from left to right, i.e., the central tank is the tank tagged as 3 . There exist two sensors measuring the liquid level, $h$, in $T_{1}$ and $T_{2}$ with the assumption of $h_{1}>h_{3}>h_{2}$. Moreover, there are two pumps as actuators to supply the tanks $T_{1}$ and $T_{2}$ with water. Since the sensor measurement output $y_{k}$ is transferred into the remote estimator/controller and also the control signal $u_{k}$ is sent to the local TTS through the network, not only must these signals be quantized before transmission but they may also suffer from DoS attacks and UBB deception attacks compromised CP and SC communication channels, respectively.

As discussed in [50], the dynamics of TTS can be linearized around its equilibrium point $x^{*}=[0.3182,0.1517,0.2314]^{T}$, which represents the steady liquid levels in meter, and then discretized with a sampling period $h=1 \mathrm{~s}$. Thus, the system parameters in (22) are given as

$$
\begin{aligned}
A_{k} & =\left[\begin{array}{lll}
0.9889 & 0.0001 & 0.0110 \\
0.0001 & 0.9774 & 0.0119 \\
0.0110 & 0.0119 & 0.9770
\end{array}\right] \\
B_{k} & =\left[\begin{array}{cc}
64.5993 & 0.0015 \\
0.0015 & 64.2236 \\
0.3604 & 0.3910
\end{array}\right] .
\end{aligned}
$$

The other system matrices in (22) and (25) are chosen as

$$
\begin{aligned}
A_{k}^{r} & =\left[\begin{array}{ccc}
-0.1093 & 0.0001 & -0.2474 \\
0.0001 & -0.1080 & -0.2707 \\
0.0049 & 0.0053 & 0.9738
\end{array}\right], B_{k}^{w}=\left[\begin{array}{l}
0.3 \\
0.4 \\
0.1
\end{array}\right] \\
C_{k} & =\left[\begin{array}{lll}
1 & 0 & 0 \\
0 & 1 & 0
\end{array}\right], D_{k}=\left[\begin{array}{l}
1 \\
1
\end{array}\right] .
\end{aligned}
$$

The three state variables in $x_{k}$ are the liquid levels $h_{1}, h_{2}$, and $h_{3}$, respectively. In the simulation, the initial conditions of (22), (23), and (25) are taken as $x_{0}=[0.28,0.08,0.18]^{T}$, $\hat{x}_{0}=[0.271,0.074,0.17]^{T}$, and $x_{0}^{r}=[0.3,0.1,0.2]^{T}$. Suppose that the UBB noises are $w_{k}=0.02 \sin (20 k)$ and $v_{k}=$ 


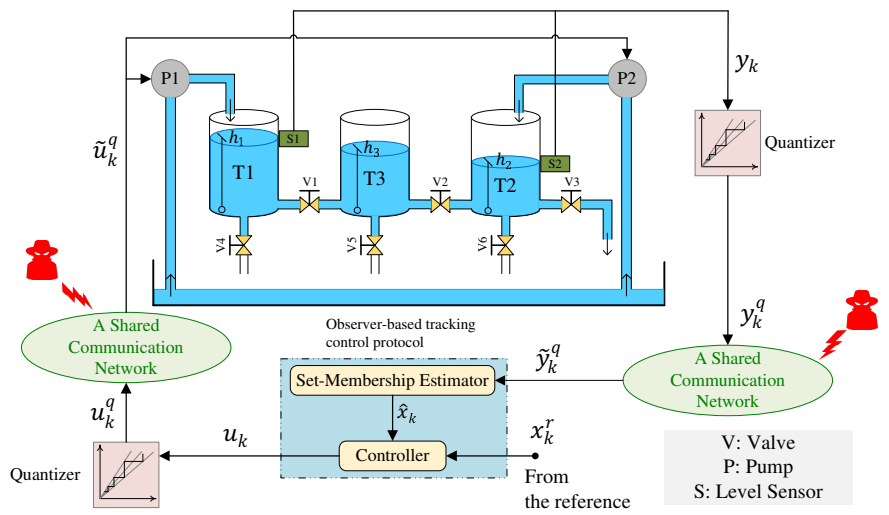

Fig. 2. Schematic of the ITTS with the developed resilient observer-based tracking control strategy.

$0.01 \cos (2 k)$ and set $W_{k}=V_{k}=0.001$. Then it can be easily checked that $w_{k}$ and $v_{k}$ belong to the ellipsoidal sets defined in (3) and (4), respectively. Set $R_{0}=\operatorname{diag}\{40,40,40\}$ and $P_{0}=\{30,30,30\}$. Furthermore, the parameters of the logarithmic quantizers are taken as $\mu_{\ell, 0}^{u}=\mu_{\ell, 0}^{y}=3, \rho_{\ell}^{u}=0.5$, and $\rho_{\ell}^{y}=0.7$. Then it yields that $H_{1}^{u}=0.67, H_{2}^{u}=1.33$, $H_{1}^{y}=0.8235$, and $H_{2}^{y}=1.1765$. Due to the fluctuation of liquid surface caused by the inflow from the pumps, the liquid level may be biased by up to $0.03 \mathrm{~m}$ around the equilibrium point in each tank [50]. Therefore, it is expected from the developed resilient tracking controller to reach a tracking error less than the bias value.

For a DoS attack on the CP communication channels, it is assumed that the lower- and upper-bounds of the unknown model parameter matrix $\Gamma_{k}=\operatorname{diag}\left\{\gamma_{1, k}, \gamma_{2, k}\right\}$ in (10) are as $\operatorname{diag}\{0,0.1\} \leq \Gamma_{k} \leq \operatorname{diag}\{0.2,0.4\}$. One can see from the DoS attack model parameter matrix that the jamming status on the channel transmitting the first component of the control signal $u_{k}^{1}$ is more severe than the one compromising the channel of the second component $u_{k}^{2}$ since the control signal $u_{k}^{1}$ may be completely lost due to successful attack on its channel. In the simulation, it is considered that the attacker performs the DoS attack from $k=1$ till $k=15$.

In the case of a UBB deception attack on the SC communication channels, suppose that the physical constraint matrix $\Lambda_{k}=\operatorname{diag}\left\{\lambda_{1, k}, \lambda_{2, k}\right\}$ is characterized by $\operatorname{diag}\{0.2,0.6\} \leq$ $\Lambda_{k} \leq \operatorname{diag}\{1.3,1.3\}$. From the lower- and upper-bounds on the physical constraints affecting the UBB deception attack signal, it can be seen that the attack on the tank $T_{2}$ sensor's measurement output is less degraded by the imposed physical constraints than the one violating the tank $T_{1}$ sensor's measurement output. In the simulation, the UBB deception attack is launched by the attacker from $k=10$ till $k=50$. The UBB deception attack signal is assumed to be $\delta_{k}=$ $[0.07 \sin (k), 0.08 \cos (k)]^{T}$ whose entries represent the attacks on the SC channels transmitting the measurement outputs of tanks $T_{1}$ and $T_{2}$, respectively.

Fig. 3 depicts the liquid level in each tank when the tracking controller and estimator are not resilient against the attacks, i.e., tracking control and estimation strategies are designed without any prior consideration of the existence of attacks. Since the attacker perpetrates the DoS attack on the CP communication channels so as to prevent the tracking control commands $u_{1}$ and $u_{2}$ from transferring to tanks $T_{1}$ and $T_{2}$,

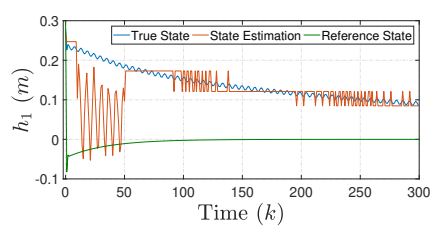

(a)

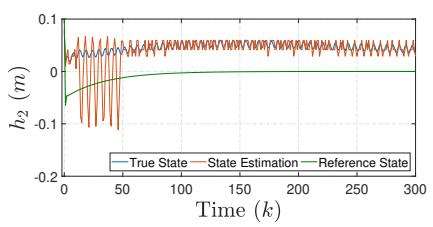

(b)

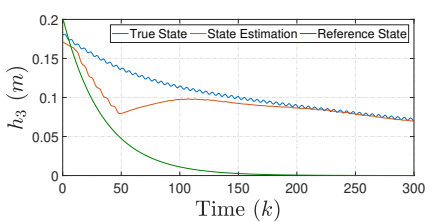

(c)

Fig. 3. Non-resilient tracking control and estimation performance on liquid levels (a) $h_{1}$; (b) $h_{2}$; and (c) $h_{3}$.

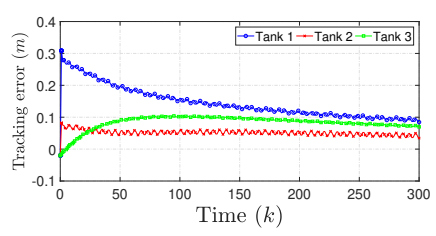

(a)

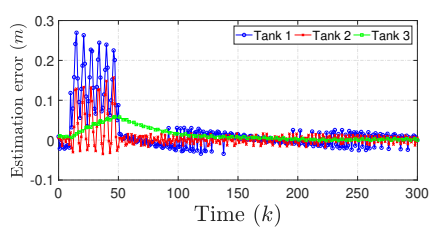

(b)
Fig. 4. (a) Non-resilient tracking error. (b) Non-resilient estimation error.

respectively, the liquid levels in the three tanks of ITTS fail to track their reference signals. Furthermore, due to the existence of the deception attack on the SC communication channels, the estimator receives the corrupted measurement outputs. The simulated deception attack affects the amplitude and frequency of the signal measured by each sensor and hence, large fluctuations occur on the state estimation at each tank causing the estimation deviates from the true state significantly. As a result of the attacks on both $\mathrm{CP}$ and SC communication channels, tracking error, $x_{k}-x_{k}^{r}$, increases dramatically with the maximum of $0.3 \mathrm{~m}$ in tank $T_{1}$ and $0.1 \mathrm{~m}$ in tanks $T_{2}$ and $T_{3}$ as shown in Fig. 4a. Additionally, as demonstrated in Fig. $4 \mathrm{~b}$, the attack makes the estimation error, $x_{k}-\hat{x}_{k}$ rise by the maximum of $0.28 \mathrm{~m}, 0.15 \mathrm{~m}$, and $0.05 \mathrm{~m}$ in tanks $T_{1}, T_{2}$, and $T_{3}$, respectively, which are considerably above the predetermined value of the bias, i.e., $0.03 \mathrm{~m}$.

Let us carry out the same attacks on $\mathrm{CP}$ and SC communication channels while considering the developed resilient setmembership tracking control and estimation methods. As illustrated in Fig. 5, the liquid level in each tank can perfectly track the reference signal although the availability of the control commands $u_{1}$ and $u_{2}$ and the integrity of the measurement outputs $y_{1}$ and $y_{2}$ are compromised by the DoS attack on the CP channels and the deception attack on the SC channels, respectively. More specifically, when these attacks are present, the two-step set-membership estimator adjusts the gains $\hat{A}_{k}$ and $\hat{B}_{k+1}$ in (23) such that deviation of the state estimation from the true state is minimized and thus, the prediction and estimation errors are constrained by the predefined bounding ellipsoidal sets in (26) and (27). The secure state estimation is employed by the observer-based tracking control protocol (24) in order to generate the tracking control command. However, the control signal is subject to be corrupted by the DoS 


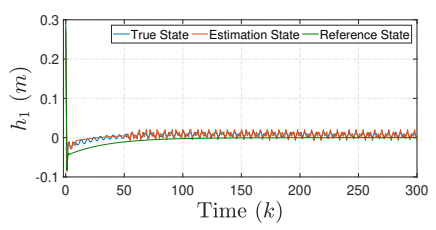

(a)

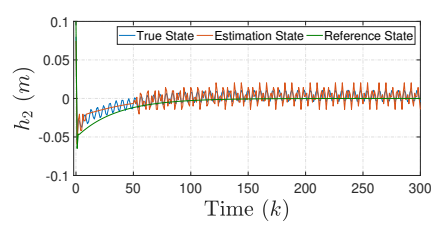

(b)

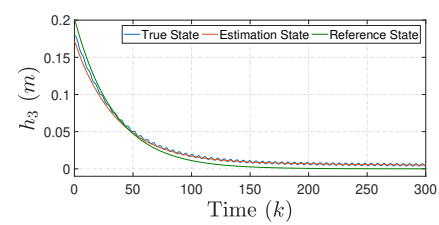

(c)

Fig. 5. Resilient tracking control and estimation performance on liquid levels (a) $h_{1}$; (b) $h_{2}$; and (c) $h_{3}$.

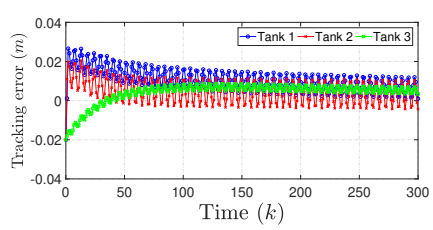

(a)

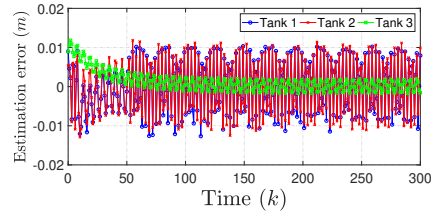

(b)
Fig. 6. (a) Resilient tracking error. (b) Resilient estimation error.

attack on CP communication channels. Therefore, the tracking controller modifies the gain $M_{k}$ in the proposed protocol (24) at every instant of time so that the tracking error is constrained by the predefined bounding ellipsoidal set in (28). Therefore, the proposed resilient set-membership tracking control and estimation methods can lead to a significant reduction in the tracking and estimation errors compared to the non-resilient case. As shown in Fig. 6a, the maximum tracking error drops approximately into $0.025 \mathrm{~m}$ in tank $T_{1}$ and $0.02 \mathrm{~m}$ in tanks $T_{2}$, and $T_{3}$, respectively, which are less than the predetermined value of the bias, i.e., $0.03 \mathrm{~m}$. Furthermore, from Fig. 6b, the maximum estimation error decreases to about $0.01 \mathrm{~m}$ in each tank.

\section{CONCLUSiON}

The problem of resilient tracking control in an NCS subject to two different attacks, UBB process noise and measurement noise, and limited communication capacity has been addressed. More specifically, a DoS attack has been considered on the communication channels between a remote controller and a physical plant, where the adversary violates the availability of the tracking control command. In addition, the adversary compromises the integrity of the communication channels between a sensor and a remote controller by launching a deception attack on those channels. Furthermore, due to limited communication capacity in real-world application of wireless networks, it has been considered that the signals are quantized by a logarithmic quantizer prior to enter the communication networks. The concept of resilient setmembership tracking control technique has been introduced. Also, a resilient two-step set-membership estimator is provided to secure the state estimation against those attacks. To solve out the parameters of the resilient tracking controller and the resilient estimator, a convex optimization algorithm has been proposed by recursively computing of confidence reference state ellipsoid and state estimation ellipsoids, which contain the true state of the system. The effectiveness and applicability of the derived results have been verified via an internet-based three-tank system. It should be mentioned that when modeling cyber attacks on the $\mathrm{CP}$ and SC communication channels, one may take into account a combination of deception attacks and DoS attacks to form a sophisticated and stealthy attack model and therefore, it constitutes one of our future research work. Furthermore, this study can be further developed by considering the resource-efficient issue in NCSs subject to the presence of cyber attacks and limited communication resources. The exiting event-triggered control techniques provide some useful insights for the design of the desired resilient event-triggered tracking control methods for this class of NCSs.

\section{APPENDIX}

Proof of Theorem 1: The proof is twofold.

1) Proof of resilient set-membership tracking control. At time $k$, if $\left(x_{k}-x_{k}^{r}\right)^{T} R_{k}^{-1}\left(x_{k}-x_{k}^{r}\right) \leq 1$, by Schur complement, the inequality is equivalent to $\left(x_{k}-x_{k}^{r}\right)\left(x_{k}-x_{k}^{r}\right)^{T} \leq R_{k}$. From Cholesky factorization, one has $R_{k}=L_{k} L_{k}^{T}$. Let $\vartheta_{1}=$ $L_{k}^{-1}\left(x_{k}-x_{k}^{r}\right)$, then we have

$$
\vartheta_{1}^{T} \vartheta_{1}=\left(x_{k}-x_{k}^{r}\right)^{T} R_{k}^{-1}\left(x_{k}-x_{k}^{r}\right) \leq 1
$$

which means that there is a vector $\vartheta_{1}$ satisfying $\left\|\vartheta_{1}\right\| \leq 1$ such that $x_{k}=x_{k}^{r}+L_{k} \vartheta_{1}$.

For brevity, we denote $\sigma_{k}^{u}=\sigma^{u}\left(u_{k}\right)$ and $\bar{u}_{k}=\tilde{\Gamma}_{k} u_{k}^{q}$. Then, the one-step ahead state tracking error can be calculated as

$$
x_{k+1}-x_{k+1}^{r}=\Pi_{k}^{c} \eta_{k}^{c}
$$

where $\eta_{k}^{c}=\left[\begin{array}{lllll}1 & \vartheta_{1}^{T} & w_{k}^{T} & \bar{u}_{k}^{T} & \sigma_{k}^{u^{T}}\end{array}\right]^{T}$ and $\Pi_{k}^{c}$ is defined in (31). Thus, the condition in (28) can be rewritten as

$$
\eta_{k}^{c^{T}}\left(\Delta^{c}+\Pi_{k}^{c^{T}} R_{k+1}^{-1} \Pi_{k}^{c}\right) \eta_{k}^{c} \leq 0
$$

where $\Delta^{c}=-\operatorname{diag}\{1,0,0,0,0\}$.

By virtue of the elementary inequality $2 a^{T} b \leq a^{T} a+b^{T} b$, (14), and (22), one can conclude that

$$
\bar{u}_{k}^{T} \bar{u}_{k} \leq 2\left(u_{k}^{T} H_{1}^{u^{T}} \check{\Gamma}_{k}^{2} H_{k}^{u} u_{k}+\sigma_{k}^{u^{T}} \check{\Gamma}_{k}^{2} \sigma_{k}^{u}\right)
$$

where $\check{\Gamma}_{k}^{2}=\check{\Gamma}_{k}^{T} \check{\Gamma}_{k}$ since $\check{\Gamma}_{k}$ is a diagonal matrix. From (3) and (35), it is clear that $w_{k}$ and $\vartheta_{1}$ satisfy

$$
\left\{\begin{array} { l } 
{ w _ { k } ^ { T } W _ { k } ^ { - 1 } w _ { k } \leq 1 } \\
{ \| \vartheta _ { 1 } \| \leq 1 }
\end{array} \Longleftrightarrow \left\{\begin{array}{l}
\eta_{k}^{c^{T}} \Xi_{k}^{c, 1} \eta_{k}^{c} \leq 0 \\
\eta_{k}^{c^{T}} \Xi_{k}^{c, 2} \eta_{k}^{c} \leq 0
\end{array}\right.\right.
$$

where $\Xi_{k}^{c, 1}=\operatorname{diag}\left\{-1,0, W_{k}^{-1}, 0,0\right\}$ and $\Xi_{k}^{c, 2}=\operatorname{diag}\{-1, I$, $0,0,0\}$.

On the other hand, the constraint in (38) can be written in $\eta_{k}^{c}$ as

$$
\eta_{k}^{c^{T}} \Omega_{k}^{c, 1} \eta_{k}^{c} \leq 0
$$

where $\Omega_{k}^{c, 1}=\operatorname{diag}\left\{-2\left(\hat{x}_{k}-x_{k}^{r}\right)^{T} M_{k}^{T} H_{1}^{u T} \check{\Gamma}_{k}^{2} H_{k}^{u} M_{k}\left(\hat{x}_{k}-\right.\right.$ $\left.\left.x_{k}^{r}\right), 0,0, I,-2 \check{\Gamma}_{k}^{2}\right\}$. Furthermore, substituting $u_{k}$ from (24) into (9) yields

$$
\sigma_{k}^{u^{T}}\left(\sigma_{k}^{u}-H_{k}^{u} M_{k}\left(\hat{x}_{k}-x_{k}^{r}\right)\right) \leq 0
$$

which can be expressed in $\eta_{k}^{c}$ as

$$
\eta_{k}^{c^{T}} \Omega_{k}^{c, 2} \eta_{k}^{c} \leq 0
$$

where $\Omega_{k}^{c, 2}=\left[\Omega_{\alpha, \beta}^{c, 2}(k)\right]_{5 \times 5}$ is a time-varying sparse symmetric block matrix whose nonzero entries given by $\Omega_{1,5}^{c, 2}(k)=$ $-\Theta_{1,5}^{c}(k), \Omega_{5,5}^{c, 2}(k)=-\Theta_{5,5}^{c}(k)+2 \check{\Gamma}_{k}^{2}$. 
Applying $\mathcal{S}$-procedure, (37) holds if there exist positive scalar sequences $\tau_{m, k}, m=1,2$, such that

$\Pi_{k}^{c^{T}} R_{k+1}^{-1} \Pi_{k}^{c}+\Delta^{c}-\Omega_{k}^{c, 1}-\Omega_{k}^{c, 2}-\tau_{1, k} \Xi_{k}^{c, 2}-\tau_{2, k} \Xi_{k}^{c, 1} \leq 0$.

Applying Schur complement to (42) straightforwardly gets the right matrix inequality in (31). This completes the proof.

2) Proof of resilient set-membership state estimation at the predicted state estimate step. The proof is similar to that of Theorem 1, part 1, and is therefore omitted due to the page limit.

Proof of Theorem 2: resilient set-membership state estimation at the updated state estimate step. By Theorem 1, if $x_{k+1}$ belongs to $\left(x_{k+1}-\hat{x}_{k+1 \mid k}\right)^{T} P_{k+1 \mid k}^{-1}\left(x_{k+1}-\hat{x}_{k+1 \mid k}\right) \leq 1$, then there exists a vector $\vartheta_{3}$ satisfying

$$
\vartheta_{3}^{T} \vartheta_{3}=\left(x_{k+1}-\hat{x}_{k+1 \mid k}\right)^{T} P_{k+1 \mid k}^{-1}\left(x_{k+1}-\hat{x}_{k+1 \mid k}\right) \leq 1
$$

such that $x_{k+1}=\hat{x}_{k+1 \mid k}+E_{k+1 \mid k} \vartheta_{3}$, where $E_{k+1 \mid k}$ is a factorization of $P_{k+1 \mid k}=E_{k+1 \mid k} E_{k+1 \mid k}^{T}$. By denoting $\sigma^{y}\left(y_{k}\right)=\sigma_{k}^{y}$ and $\psi\left(y_{k}^{q, a}\right)=\psi_{k}$, the one-step ahead state estimation error can be obtained as

$$
x_{k+1}-\hat{x}_{k+1}=\Pi_{k}^{e} \eta_{k}^{e}
$$

where $\eta_{k}^{e}=\left[\begin{array}{llllll}1 & \vartheta_{3}^{T} & v_{k+1}^{T} & \delta_{k+1}^{T} & \sigma_{k+1}^{y^{T}} & \psi_{k+1}^{T}\end{array}\right]^{T}$ and $\Pi_{k}^{e}$ is defined in (32). Hence, the condition in (27) can be rewritten as

$$
\eta_{k}^{e^{T}}\left(\Delta^{e}+\Pi_{k}^{e^{T}} P_{k+1}^{-1} \Pi_{k}^{e}\right) \eta_{k}^{e} \leq 0
$$

where $\Delta^{e}=-\operatorname{diag}\{1,0,0,0,0,0\}$.

From (4), (16), and (43), we have the following constraints

$$
\left\{\begin{array} { l } 
{ v _ { k + 1 } ^ { T } V _ { k + 1 } ^ { - 1 } v _ { k + 1 } \leq 1 } \\
{ \delta _ { k + 1 } ^ { T } S _ { k + 1 } ^ { - 1 } \delta _ { k + 1 } \leq 1 } \\
{ \| \vartheta _ { 3 } \| \leq 1 }
\end{array} \Longleftrightarrow \left\{\begin{array}{l}
\eta_{k}^{e^{T}} \Xi_{k}^{e, 1} \eta_{k}^{e} \leq 0 \\
\eta_{k}^{e^{T}} \Xi_{k}^{e, 2} \eta_{k}^{e} \leq 0 \\
\eta_{k}^{e^{T}} \Xi_{k}^{e, 3} \eta_{k}^{e} \leq 0
\end{array}\right.\right.
$$

where $\Xi_{k}^{e, 1}=\operatorname{diag}\left\{-1,0, V_{k+1}^{-1}, 0,0,0\right\}, \Xi_{k}^{e, 2}=\operatorname{diag}\{-1,0$, $\left.0, S_{k+1}^{-1}, 0,0\right\}$, and $\Xi_{k}^{e, 3}=\operatorname{diag}\{-1, I, 0,0,0,0\}$.

From (22) and noting that $x_{k+1}=\hat{x}_{k+1 \mid k}+E_{k+1 \mid k} \vartheta_{3}$, it can be easily obtained that

$$
\begin{aligned}
& y_{k+1}=C_{k+1}\left(\hat{x}_{k+1 \mid k}+E_{k+1 \mid k} \vartheta_{3}\right)+D_{k+1} v_{k+1} \\
& \begin{array}{r}
y_{k+1}^{q, a}=-H_{1}^{y} C_{k+1}\left(\hat{x}_{k+1 \mid k}+E_{k+1 \mid k} \vartheta_{3}\right) \\
\quad-H_{1}^{y} D_{k+1} v_{k+1}-\sigma_{k+1}^{y}+\delta_{k+1} .
\end{array}
\end{aligned}
$$

Substituting (47) into (9) and also inserting (48) into (20), respectively, yield

$$
\left\{\begin{array}{c}
\sigma_{k+1}^{y^{T}}\left(\sigma_{k+1}^{y}-H^{y} C_{k+1}\left(\hat{x}_{k+1 \mid k}+E_{k+1 \mid k} \vartheta_{3}\right)\right. \\
\left.-H^{y} D_{k+1} v_{k+1}\right) \leq 0 \\
\psi_{k+1}^{T}\left(\psi_{k+1}-\tilde{\Lambda}_{k+1}\left(-H_{1}^{y} C_{k+1}\left(\hat{x}_{k+1 \mid k}+E_{k+1 \mid k} \vartheta_{3}\right)\right.\right. \\
\left.\left.-H_{1}^{y} D_{k+1} v_{k+1}-\sigma_{k+1}^{y}+\delta_{k+1}\right)\right) \leq 0
\end{array}\right.
$$

which can be written in $\eta_{k}^{e}$ as

$$
\eta_{k}^{e^{T}} \Omega_{k}^{e} \eta_{k}^{e} \leq 0
$$

where $\Omega_{k}^{e}=\left[\Omega_{\alpha, \beta}^{e}(k)\right]_{6 \times 6}$ is a time-varying sparse symmetric block matrix with its nonzero entries given by

$$
\begin{aligned}
& \Omega_{1,5}^{e}(k)=-(1 / 2) \hat{x}_{k+1 \mid k}^{T} C_{k+1}^{T} H^{y} \\
& \Omega_{1,6}^{e}(k)=(1 / 2) \hat{x}_{k+1 \mid k}^{T} C_{k+1}^{T} H_{1}^{y} \tilde{\Lambda}_{k+1} \\
& \Omega_{2,5}^{e}(k)=-(1 / 2) E_{k+1 \mid k}^{T} C_{k+1 \mid k}^{T} H^{y}
\end{aligned}
$$

$$
\begin{aligned}
& \Omega_{2,6}^{e}(k)=(1 / 2) E_{k+1 \mid k}^{T} C_{k+1 \mid k}^{T} H_{1}^{y} \tilde{\Lambda}_{k+1} \\
& \Omega_{3,5}^{e}(k)=-(1 / 2) D_{k+1}^{T} H^{y}, \Omega_{3,6}^{e}(k)=(1 / 2) D_{k+1}^{T} H_{1}^{y} \tilde{\Lambda}_{k+1} \\
& \Omega_{4,6}^{e}(k)=-(1 / 2) \Lambda_{k+1}, \Omega_{5,5}^{e}(k)=\Omega_{6,6}^{e}(k)=I \\
& \Omega_{5,6}^{e}(k)=-\Omega_{4,6}(k) .
\end{aligned}
$$

By virtue of $\mathcal{S}$-procedure, (45) holds if there exist positive scalar sequences $\tau_{m, k}, m=5,6, \ldots, 8$, such that

$\Pi_{k}^{e^{T}} P_{k+1}^{-1} \Pi_{k}^{e}+\Delta^{e}-\tau_{5, k} \Xi_{k}^{e, 3}-\tau_{6, k} \Xi_{k}^{e, 1}-\tau_{7, k} \Xi_{k}^{e, 2}-\tau_{8, k} \Omega_{k}^{e} \leq 0$.

By applying Schur complement and noting that $S_{k+1}=$ $\tau_{7, k} \widetilde{S}_{k+1}$, (32) can be derived from (51). This completes the proof.

\section{REFERENCES}

[1] X.-M. Zhang, Q.-L. Han, X. Ge, D. Ding, L. Ding, D. Yue, and C. Peng, "Networked control systems: A survey of trends and techniques," IEEE/CAA J. Automatica Sinica, 2019, DOI: 10.1109/JAS.2019.1911651

[2] Z.-H. Pang and G.-P. Liu, "Design and implementation of secure networked predictive control systems under deception attacks," IEEE Trans. Control Syst. Technol., vol. 20, no. 5, pp. 1334-1342, Sep. 2012.

[3] Z.-H. Pang, G.-P. Liu, D.-H. Zhou and D.-H. Sun, "Two-channel false data injection attacks against output tracking control of networked systems," IEEE Trans. Ind. Electron., vol. 63, no.5, pp. 3242-3251, May 2016.

[4] Y. Shi, J. Huang, and B. Yu, "Robust tracking control of networked control systems: Application to a networked DC motor," IEEE Trans. Ind. Electron., vol. 60, no. 12, pp. 5864-5874, Dec. 2013.

[5] M. Li and Y. Chen, "Robust tracking control of networked control systems with communication constraints and external disturbance," IEEE Trans. Ind. Electron., vol. 64, no. 5, pp. 4037-4047, May 2017.

[6] W. Ye, Z. Li, C. Yang, J. Sun, C. Su, and R. Lu, "Vision-based human tracking control of a wheeled inverted pendulum robot," IEEE Trans. Cybern., vol. 46, no. 11, pp. 2423-2434, Nov. 2016.

[7] C. Lian, X. Xu, H. Chen, and H. He, "Near-optimal tracking control of mobile robots via receding-horizon dual heuristic programming," IEEE Trans. Cybern., vol. 46, no. 11, pp. 2484-2496, Nov. 2016.

[8] J. Chen, B. Jia, and K. Zhang, "Trifocal tensor-based adaptive visual trajectory tracking control of mobile robots," IEEE Trans. Cybern., vol. 47, no. 11, pp. 3784-3798, Nov. 2017.

[9] F. Liao, J. L. Wang, and G.-H. Yang, "Reliable robust flight tracking control: an LMI approach," IEEE Trans. Control Syst. Technol., vol. 10, no. 1, pp. 76-89, Jan. 2002.

[10] S. Dai, J. Fu, and J. Zhao, "Robust reliable tracking control for a class of uncertain systems and its application to flight control," Acta Autom. Sin., vol. 32, no. 5, pp. 738-745, 2006.

[11] Y. Zhang, Q. Wang, C. Dong, and Y. Jiang, " $H_{\infty}$ output tracking control for flight control systems with time-varying delay," Chinese J. Aeronautics, vol. 26, no. 5, pp. 1251-1258, Oct. 2013.

[12] J. D. Townsend, M. Saville, S. M. Hong, and R. K. Martin, "Simulator for velocity gate pull-off electronic countermeasure techniques," in Proc. IEEE Radar Conf., Italy, May 2008, pp. 1-6.

[13] Y. Yuan, Q. Zhu, F. Sun, Q. Wang, and T. Başar, "Resilient control of cyber-physical systems against denial-of-service attacks," in Proc. Int. Symp. Resil. Control Sys., CA, USA, Aug. 2013, pp. 54-59.

[14] M. Zhu and S. Martínez, "On the performance analysis of resilient networked control systems under replay attacks," IEEE Trans. Autom. Control, vol. 59, no. 3, pp. 804-808, Mar. 2014.

[15] W. Zeng and M. Chow, "Resilient distributed control in the presence of misbehaving agents in networked control systems," IEEE Trans. Cybern., vol. 44, no. 11, pp. 2038-2049, Nov. 2014.

[16] D. Meng and K. L. Moore, "Studies on resilient control through multiagent consensus networks subject to disturbances," IEEE Trans. Cybern., vol. 44, no. 11, pp. 2050-2061, Nov. 2014.

[17] Y. Yuan, H. Yuan, L. Guo, H. Yang, and S. Sun, "Resilient control of networked control system under DoS attacks: A unified game approach," IEEE Trans. Ind. Informat., vol. 12, no. 5, pp. 1786-1794, Oct. 2016.

[18] Y. Yuan, H. Yuan, D. W. C. Ho, and L. Guo, "Resilient control of wireless networked control system under denial-of-service attacks: A cross-layer design approach," IEEE Trans. Cybern., 2018, DOI: 10.1109/TCYB.2018.2863689.

[19] S. Hu, D. Yue, X. Xie, X. Chen, and X. Yin, "Resilient event-triggered controller synthesis of networked control systems under periodic DoS jamming attacks," IEEE Trans. Cybern., vol. 49, no. 12, pp. 4271-4281, Dec. 2019 
[20] R. Moghadam and H. Modares, "Resilient autonomous control of distributed multiagent systems in contested environments," IEEE Trans. Cybern., vol. 49, no. 11, pp. 3957-3967, Nov. 2019.

[21] H. Yuan, Y. Xia, H. Yang, and Y. Yuan, "Resilient control for wireless networked control systems under DoS attack via a hierarchical game," Int. J. Robust Nonlin. Control, vol. 28, no. 15, pp. 4604-4623, 2018.

[22] Y. Wan and J. Cao, "Observer-based tracking control for heterogeneous dynamical systems under asynchronous attacks," in Proc. Int. Workshop Complex Sys. Netw., Doha, Qatar, Dec. 2017, pp. 224-229.

[23] Z. Feng, G. Wen, and G. Hu, "Distributed secure coordinated control for multiagent systems under strategic attacks," IEEE Trans. Cybern., vol. 47, no. 5, pp. 1273-1284, May 2017.

[24] Y. Tang, D. Zhang, D. W. C. Ho, W. Yang, and B. Wang, "Event-based tracking control of mobile robot with denial-of-service attacks," IEEE Trans. Syst., Man, Cybern., Syst., 2018, DOI: 10.1109/TSMC.2018.2875793.

[25] H. Khalil, Nonlinear Systems. 2nd ed., Upper Saddle River, NJ: PrenticeHall, 1996.

[26] L. Ghaoui and G. Calafiore, "Robust filtering for discrete-time systems with bounded noise and parameteric uncertainty," IEEE Trans. Autom. Control, vol. 46, no. 7, pp. 1084-1089, Jul. 2001.

[27] X. Ge, Q.-L. Han, and F. Yang, "Event-based set-membership leaderfollowing consensus of networked multi-agent systems subject to limited communication resources and unknown-but-bounded noise," IEEE Trans. Ind. Electron., vol. 64, no. 6, pp. 5045-5054, Jun. 2017.

[28] X. Ge, Q.-L. Han, and Z. Wang, "A dynamic event-triggered transmission scheme for distributed set-membership estimation over wireless sensor networks," IEEE Trans. Cybern., vol. 49, no. 1, pp. 171-183, Jan. 2019.

[29] M. Fu and L. Xie, "The sector bound approach to quantized feedback control," IEEE Trans. Autom. Control, vol. 50, no. 11, pp. 1698-1711, Nov. 2005.

[30] N. Elia and S. K. Mitter, "Stabilization of linear systems with limited information," IEEE Trans. Autom. Control, vol. 46, no. 9, pp. 1384-1400, Sep. 2001.

[31] M. Fu and L. Xie, "Finite-level quantized feedback control for linear systems," IEEE Trans. Autom. Control, vol. 54, no. 5, pp. 1165-1170, May 2009.

[32] L. Ma, Z. Wang, Q.-L. Han, and H. Lam, "Variance-constrained distributed filtering for time-varying systems with multiplicative noises and deception attacks over sensor networks," IEEE Sensors J., vol. 17, no. 7, pp. 2279-2288, Apr. 2017.

[33] D. Ding, Q.-L. Han, Z. Wang, and X. Ge, "A survey on model-based distributed control and filtering for industrial cyber-physical systems," IEEE Trans. Ind. Informat., vol. 15, no. 5, pp. 2483-2499, May 2019.

[34] Y. Mo, E. Garone, A. Casavola, and B. Sinopoli, "False data injection attacks against state estimation in wireless sensor networks," in Proc. IEEE Conf. Decis. Control, Atlanta, USA, Dec. 2010, pp. 5967-5972.

[35] Y. Li, L. Shi, P. Cheng, J. Chen, and D. E. Quevedo, "Jamming attacks on remote state estimation in cyber-physical systems: A game-theoretic approach," IEEE Trans. Autom. Control, vol. 60, no. 10, pp. 2831-2836, Oct. 2015.

[36] S. Mishra, Y. Shoukry, N. Karamchandani, S. N. Diggavi, and P. Tabuada, "Secure state estimation against sensor attacks in the presence of noise," IEEE Trans. Control Netw. Syst., vol. 4, no. 1, pp. 49-59, Mar. 2017.

[37] N. Forti, G. Battistelli, L. Chisci, S. Li, B. Wang, and B. Sinopoli, "Distributed joint attack detection and secure state estimation," IEEE Trans. Signal Inf. Process. Netw., vol. 4, no. 1, pp. 96-110, Mar. 2018.

[38] L. Zhang, Y. Zhu, Z. Ning, and X. Yin, "Resilient estimation for networked systems with variable communication capability," IEEE Trans. Autom. Control, vol. 61, no. 12, pp. 4150-5156, Dec. 2016.

[39] X. Ge, Q.-L. Han, M. Zhong, and X.-M. Zhang, "Distributed Krein space-based attack detection over sensor networks under deception attacks," Automatica, vol. 109, 108557, Nov. 2019.

[40] Y. Nakahira and Y. Mo, "Attack-resilient $H_{2}, H_{\infty}$, and $\ell_{1}$ state estimator," IEEE Trans. Autom. Control, vol. 63, no. 12, pp. 4353-4360, Dec. 2018.

[41] S. Xiao, Q.-L. Han, X. Ge, and Y. Zhang, "Secure distributed finite-time filtering for positive systems over sensor networks under deception attacks," IEEE Trans. Cybern., 2019, DOI: 10.1109/TCYB.2019.2900478.

[42] A. B. Kurzhanski and I. Valyi, Ellipsoidal calculus for estimation and control. 1st ed., Boston, MA: Birkhäuser Basel, 1997.

[43] F. C. Schweppe, "Recursive state estimation: Unknown but bounded errors and system inputs," IEEE Trans. Autom. Control, vol. 13, no. 1, pp. 22-28, Feb. 1968.
[44] D. Bertsekas and I. Rhodes, "Recursive state estimation for a setmembership description of uncertainty," IEEE Trans. Autom. Control, vol. 16, no. 2, pp. 117-128, Apr. 1971.

[45] F. Yang and Y. Li, "Set-membership filtering for systems with sensor saturation," Automatica, vol. 45, no. 8, pp. 1896-1902, Aug. 2009.

[46] F. Yang and Y. Li, "Set-membership filtering with state constraints," IEEE Trans. Aerosp. Electron. Syst., vol. 45, no. 4, pp. 1619-1629, Oct. 2009.

[47] Z. Wu, F. Yang, and Q.-L. Han, "A novel islanding fault detection for distributed generation systems," Int. J. Robust Nonlin. Control, vol. 24, no. 8-9, pp. 1431-1445, 2014.

[48] Y. Ashibani and Q. H. Mahmoud, "Cyber physical systems security: Analysis, challenges and solutions," Comput. Secur., vol. 68, pp. 81-97, Jul. 2017.

[49] L. Vandenberghe and S. Boyd, "Semidefinite programming," SIAM Rev., vol. 38, no. 1, pp. 49-95, Mar. 1996.

[50] X. He, Z. Wang, Y. Liu, L. Qin, and D. Zhou, "Fault-tolerant control for an internet-based three-tank system: accommodation to sensor bias faults," IEEE Trans. Ind. Electron., vol. 64, no. 3, pp. 2266-2275, Mar. 2017.

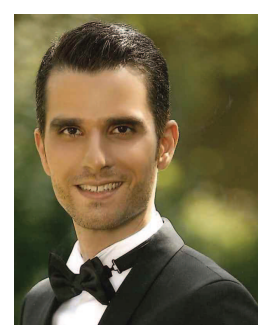

Eman Mousavinejad (S'14) received the B.Sc. degree in mechanical engineering from Azad University, Tehran, Iran, in 2006, the M.Sc. degree in mechatronics engineering (control) from the Sharif University of Technology, Tehran, Iran, in 2009, and the M.Phil. degree in mechatronics engineering (control) from Griffith University, Gold Coast, QLD, Australia, in 2014, where he is currently pursuing the Doctor of Philosophy degree in mechatronics engineering (control) with the Griffith School of Engineering.

His current research interests include networked control and filtering, multiagent systems, cyber security, vehicle dynamics and control, autonomous vehicles, drive assistance systems, by-wire systems, and linear and nonlinear systems.

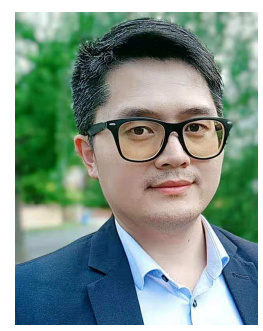

Xiaohua Ge (M'18) received the B.Eng. degree in electronics and information engineering from Nanchang Hangkong University, Nanchang, China, in 2008, the M.Eng. degree in control theory and control engineering from Hangzhou Dianzi University, Hangzhou, China, in 2011, and the Ph.D. degree in computer engineering from Central Queensland University, Rockhampton, QLD, Australia, in 2014.

From 2011 to 2013, he was a Research Assistant with the Centre for Intelligent and Networked Systems, Central Queensland University, Rockhampton, QLD, Australia, where he was a Research Fellow in 2014. From 2015 to 2017, he was a Research Fellow with the Griffith School of Engineering, Griffith University, Gold Coast, QLD, Australia. He is currently a Lecturer with the School of Software and Electrical Engineering, Swinburne University of Technology, Melbourne, VIC, Australia.

His research interests include distributed estimation over sensor networks, distributed coordination in multi-agent systems, security and privacy preserving in cyber-physical systems. 


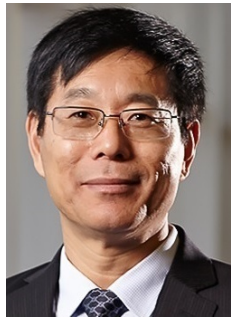

Qing-Long Han (M'09-SM'13-F'19) received the B.Sc. degree in Mathematics from Shandong Normal University, Jinan, China, in 1983, and the M.Sc. and $\mathrm{Ph} . \mathrm{D}$. degrees in Control Engineering and Electrical Engineering from East China University of Science and Technology, Shanghai, China, in 1992 and 1997, respectively.

From September 1997 to December 1998, he was a Post-doctoral Researcher Fellow with the Laboratoire d'Automatique et d'Informatique Industielle (currently, Laboratoire d'Informatique et d'Automatique pour les Systémes), École Supérieure d'Ing'enieurs de Poitiers (currently, École Nationale Supérieure d'Ingé nieurs de Poitiers), Université de Poitiers, France. From January 1999 to August 2001, he was a Research Assistant Professor with the Department of Mechanical and Industrial Engineering at Southern Illinois University at Edwardsville, USA. From September 2001 to December 2014, he was Laureate Professor, an Associate Dean (Research and Innovation) with the Higher Education Division, and the Founding Director of the Centre for Intelligent and Networked Systems at Central Queensland University, Australia. From December 2014 to May 2016, he was Deputy Dean (Research), with the Griffith Sciences, and a Professor with the Griffith School of Engineering, Griffith University, Australia. In May 2016, he joined Swinburne University of Technology, Australia, where he is currently Pro Vice-Chancellor (Research Quality) and a Distinguished Professor. In March 2010, he was appointed Chang Jiang (Yangtze River) Scholar Chair Professor by Ministry of Education, China. His research interests include networked control systems, multi-agent systems, time-delay systems, complex dynamical systems and neural networks.

Professor Han is a Highly Cited Researcher according to Clarivate Analytics (formerly Thomson Reuters). He is a Fellow of The Institution of Engineers Australia. He is an Associate Editor of several international journals, including the IEEE TRANSACTIONS ON CYBERNETICS, the IEEE TRANSACTIONS ON INDUSTRIAL ELECTRONICS, the IEEE TRANSACTIONS ON INDUSTRIAL INFORMATICS, IEEE INDUSTRIAL ELECTRONICS MAGAZINE, the IEEE/CAA JOURNAL OF AUTOMATICA SINICA, Control Engineering Practice, and Information Sciences.

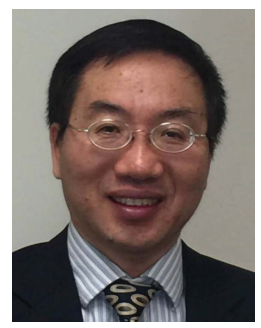

Fuwen Yang (M'99-SM'04) received the Ph.D. degree in control engineering from Huazhong University of Science and Technology, China, in 1990.

$\mathrm{He}$ was a Research Fellow with Brunel University London, Uxbridge, U.K., and King's College London, London, U.K., a Professor with Fuzhou University, Fuzhou, China, and the East China University of Science and Technology, Shanghai, China, and an Associate Professor with Central Queensland University, Rockhampton, QLD, Australia. He has also held visiting professor appointments with the University of Manchester, Manchester, U.K., and The University of Hong Kong, Hong Kong. He has published over 200 journal and conference papers. $\mathrm{He}$ is currently an Associate Professor with the Griffith School of Engineering and Built Environment, Griffith University, Gold Coast, QLD, Australia. His current research interests include networked control systems, distributed filtering and sensing, reliable fault detection and diagnosis, distributed control and filtering for smart girds, and solar PV power generation systems.

Dr. Yang is an Associate Editor of the IEEE CSS Conference Editorial Board.

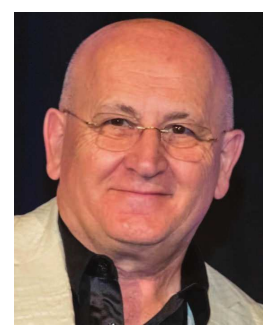

Ljubo Vlacic (M'92-SM'96) received the Diploma degree in electrical engineering and the M.Phil. and $\mathrm{Ph} . \mathrm{D}$. degrees in control systems engineering from the University of Sarajevo, Sarajevo, Bosnia and Herzegovina, in 1973, 1976, and 1986, respectively.

$\mathrm{He}$ is currently with the Institute for Integrated and Intelligent Systems, Griffith University, Gold Coast, QLD, Australia. He is also a Control Systems Scientist and a Practitioner, renowned for his contributions to co-operative driverless vehicles and intelligent control systems research and development. $\mathrm{He}$ has held a number of leading roles in both industry and academia.

Professor Emeritus Vlacic was a recipient of 19 awards, including the IEE Achievement Medal (world-wide), the Sir Lionel Hooke Award, the Queensland Professional Engineer of the Year Award, and the Gold Coast Business Events Ambassador Award. He is currently the Chair of the Board, Engineers Australia ITEE College and the IET Network Queensland and the General Chair of the 2019 IEEE Intelligent Transportation Systems Conference. He hosted ten national and international scientific conferences. His research achievements made news headlines and were broadcast through media outlets throughout the world. He is currently the Editor-in-Chief of the IEEE Intelligent Transportation Systems Magazine. 